(C) 2001 American Chemical Society, Inorg. Chem., Minkwitz ic010239w Sipporting Info Page 1 $\therefore$

SUPPLEMENTARY MATERIAL

\title{
SYNTHESIS AND CHARACTERIZATION OF THE FIRST EXAMPLES OF \\ PERFLUOROALKYL SUBSTITUTED TRIALKYL-OXONIUM SALTS,
}

$\left[\left(\mathrm{CH}_{3}\right)_{2} \mathrm{OCF}_{3}\right]^{+}\left[\mathrm{Sb}_{2} \mathrm{~F}_{11}\right]^{-} \mathrm{AND}\left[\left(\mathrm{CH}_{3}\right)_{2} \mathrm{OCF}\left(\mathrm{CF}_{3}\right)_{2}\right]^{+}\left[\mathrm{Sb}_{2} \mathrm{~F}_{11}\right]^{-}$

Rolf Minkwitz*, Stefan Reinemann, Raphael Seelbinder, Detlef Konikowski

Anorganische Chemie, Fachbereich Chemie der Universität Dortmund, D-44221 Dortmund,

Germany

Hans Hart1*, Irene Brüdgam

Institut für Anorganische und Analytische Chemie der Freien Universität Berlin, D-14195 Berlin,

Germany

Joachim Hegge, Berthold Hoge, Jeffrey A. Sheehy, Karl O. Christe*

Loker Hydrocarbon Research Institute, University of Southern California, Los Angeles, California 90089, and Propulsion Sciences and Advanced Concepts Division, Air Force Research Laboratory, Edwards Air Force Base, California 93524-7680

\section{Abstract}

In the superacidic $\mathrm{HF} / \mathrm{SbF}_{5}$ system, methyl-trifluoromethylether forms at $-78^{\circ} \mathrm{C}$ the new tertiary oxonium salt, $\left[\left(\mathrm{CH}_{3}\right)_{2} \mathrm{OCF}_{3}\right]^{+}\left[\mathrm{Sb}_{2} \mathrm{~F}_{11}\right]$, that was characterized by Raman and multinuclear NMR spectroscopy and its crystal structure. The same oxonium salt was also obtained by methylation of $\mathrm{CH}_{3} \mathrm{OCF}_{3}$ with $\mathrm{CH}_{3} \mathrm{~F}$ and $\mathrm{SbF}_{5}$ in $\mathrm{HF}$ solution at -30 to $-10^{\circ} \mathrm{C}$. Replacement of one methyl group in the trimethyloxonium cation by the bulkier and more electronegative trifluoromethyl group increases the remaining $\mathrm{O}-\mathrm{CH}_{3}$ bond lengths by $0.037(1) \AA$ and the sum of the C-O-C bond angles by about $4.5^{\circ}$. Methylation of $\mathrm{CH}_{3} \mathrm{OCF}\left(\mathrm{CF}_{3}\right)_{2}$ with $\mathrm{CH}_{3} \mathrm{~F}$ in $\mathrm{HF} / \mathrm{SbF}_{5}$ solution at $-30{ }^{\circ} \mathrm{C}$ produces $\left[\left(\mathrm{CH}_{3}\right)_{2} \mathrm{OCF}\left(\mathrm{CF}_{3}\right)_{2}\right]^{+}\left[\mathrm{Sb}_{2} \mathrm{~F}_{11}\right]$. The observed structure and vibrational and NMR spectra were confirmed by theoretical studies at the B3LYP/6-311++G(2d,2p) and the MP2/6-311++G(2d,p) levels. 
Table S1. Crystal Data and Structure Refinement for $\left[\left(\mathrm{CH}_{3}\right)_{2} \mathrm{OCF}_{3}\right]^{+}\left[\mathrm{Sb}_{2} \mathrm{~F}_{1 \mathrm{I}}\right]^{-}$

Empirical formula

Formula weight

Temperature

Wavelength

Crystal system, space group

Unit cell dimensions

Volume

Z, Calculated density

Absorption coefficient

$\mathrm{F}(000)$

Theta range for data collection Index ranges

$\therefore$ Reflections collected/unique -

Completeness to 2 theta $=30.06$

Refinement method

Data / restraints / parameters

Goodness-of-fit on $F^{2}=$

Final $R$ indices $[\mathrm{I}>2$ sigma(I)]

$\mathrm{R}$ indices (all data)

Absolute structure parameter

Extinction coefficient

Largest diff. peak and hole
C3 H6 F14 $0 \mathrm{Sb} 2$

567.58

$171(2) \mathrm{K}$

$0.71073 \mathrm{~A}$

$\mathrm{Cmc2}_{1}$ (No. 36)

$a=9.293(6)$ A alpha $=90 \mathrm{deg}$

$b=12.456(7)$ beta $=$ A $90 \mathrm{deg}$

$c=11.548(8)$ A gamma $=90 \mathrm{deg}$

$1336.7(15) \mathrm{A}^{3}$

$4,2.820 \mathrm{Mg} / \mathrm{m}^{3}$

$4.199 \mathrm{~mm}^{-1}$

1040

2.73 to $30.06 \mathrm{deg}$.

$-12 \leq h \leq 13,0 \leq k \leq 16,0 \leq 1 \leq 15$

$1659 / 907[R($ int $)=0.0383]$

$84.1 \%$

Full-matrix least-squares on $\mathrm{F}^{2}$

$907 / 1 / 107$

$-1.089<41$

$R 1=0.0225, w R 2=0.0620$

$\mathrm{R} 1=0.0231, \mathrm{wR2}=0.0624$

$0.00(6)$

$0.0023(3)$

$\therefore \quad 0.687$ and $-0.683 \mathrm{e}^{-3} \mathrm{~A}^{-3}$ 
(c) 2001 American Chemical Society, Inorg. Chem., Minkwitz ic010239w Supporting Info Page 3

Tabie S2. Atomic coordinates $\left(\times 10^{4}\right)$ and equivalent isotropic displacement parameters $\left(\mathrm{A}^{2} \times 10^{3}\right)$ for $\left[\left(\mathrm{CH}_{3}\right)_{2} \mathrm{OCF}_{3}\right]^{+}\left[\mathrm{Sb}_{2} \mathrm{~F}_{11}\right]^{-}$. U(eq) is defined as one third of the trace of the orthogonalized Uij tensor.

\begin{tabular}{lllll}
\hline & $\mathrm{x}$ & $\mathrm{y}$ & $\mathrm{z}$ & $\mathrm{U}(\mathrm{eq})$ \\
\hline $\mathrm{Sb}(1)$ & 0 & $\ddots 11005(1)$ & $0(1)$ & $31(1)$ \\
$\mathrm{Sb}(2)$ & 0 & $7860(1)$ & $248(1)$ & $30(1)$ \\
$\mathrm{F}(1)$ & 0 & $6381(4)$ & $-44(9)$ & $51(2)$ \\
$\mathrm{F}(2)$ & $1415(6)$ & $8162(4)$ & $-824(5)$ & $57(1)$ \\
$\mathrm{F}(3)$ & $-1402(7)$ & $7770(4)$ & $1393(5)$ & $65(2)$ \\
$\mathrm{F}(4)$ & 0 & $9460(4)$ & $567(7)$ & $54(2)$ \\
$\mathrm{F}(5)$ & $1952(6)$ & $10893(5)$ & $47(17)$ & $145(6)$ \\
$\mathrm{F}(6)$ & 0 & $12431(5)$ & $-478(8)$ & $60(2)$ \\
$\mathrm{F}(7)$ & 0 & $11340(8)$ & $1519(9)$ & $149(8)$ \\
$\mathrm{F}(8)$ & 0 & $10460(6)$ & $-1449(9)$ & $128(7)$ \\
$\mathrm{F}(10)$ & 0 & $3535(5)$ & $2371(8)$ & $113(5)$ \\
$\mathrm{F}(11)$ & $1128(7)$ & $4297(5)$ & $1016(5)$ & $71(2)$ \\
$\mathrm{O}(1)$ & 0 & $5322(5)$ & $2279(5)$ & $34(1)$ \\
$\mathrm{C}(2)$ & 0 & $4306(8)$ & $1670(10)$ & $44(2)$ \\
$\mathrm{C}(1)$ & $1349(9)$ & $5574(6)$ & $2951(7)$ & $50(2)$ \\
\hline
\end{tabular}


Table S3. Bond lengths $[\mathrm{A}]$ and angles $[\mathrm{deg}]$ for $\left[\left(\mathrm{CH}_{3}\right)_{2} \mathrm{OCF}_{3}\right]^{+}\left[\mathrm{Sb}_{2} \mathrm{~F}_{11}\right]$

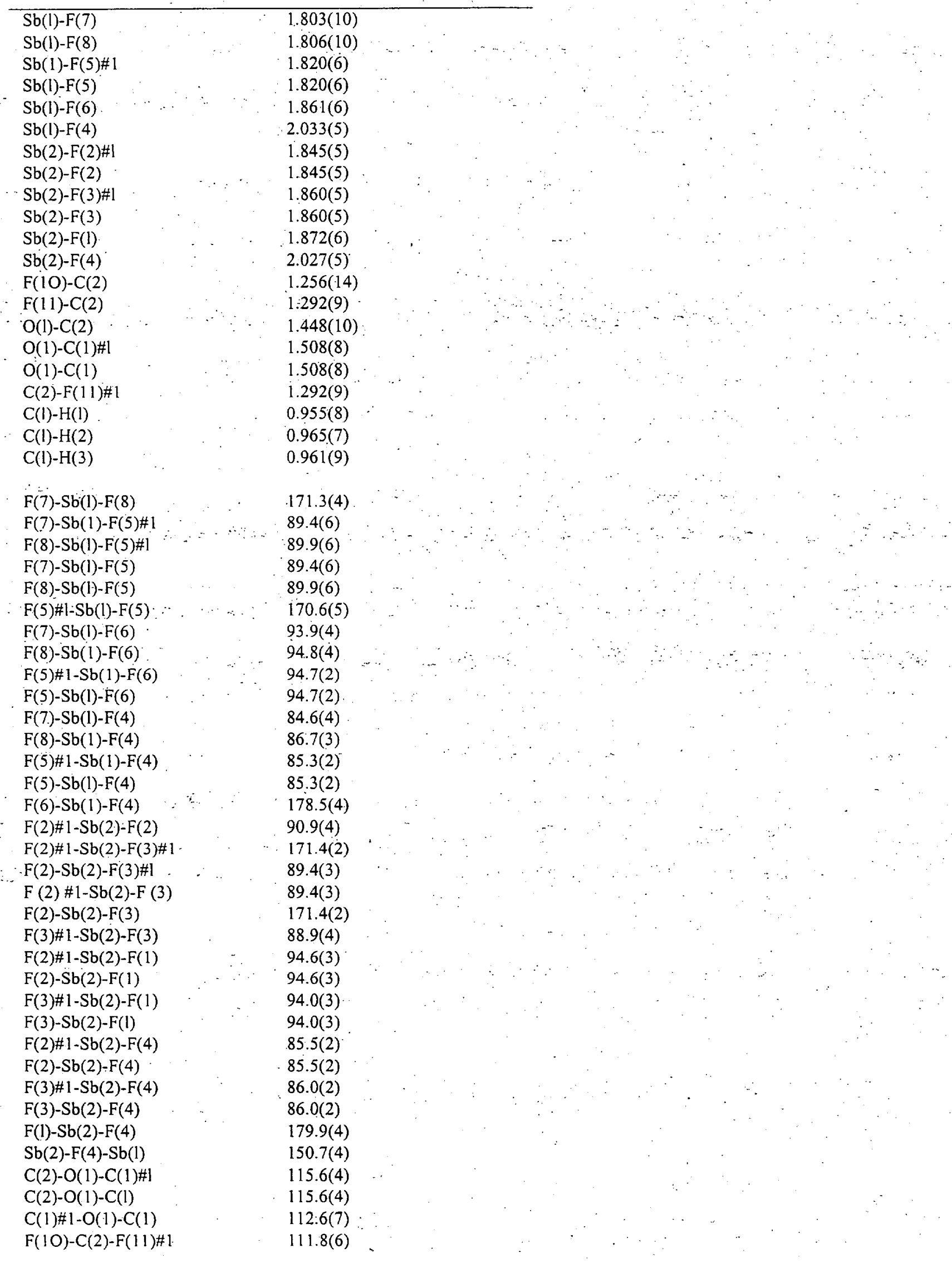


(c) 2001 American Chemical Society, Inorg. Chem., Minkwitz ic010239w Supporting Info Page 5

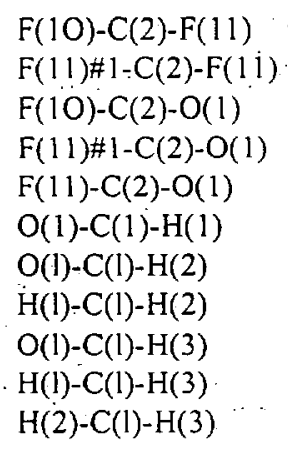

$111.8(6)$

$108.4(10)$

$110.8(9)$

$106,9(6)$

$106.9(6)$

$109.8(7)$

109.2(7)

$109.5(8)$

109.5(6)

$109.9(8)$

$109.0(8)$

Symmetry transformations used to generate equivalent atoms: \#1, - $x, y, z$ 
(c) 2001 American Chemical Society, Inorg. Chem., Minkwitz ic010239w Supporting Info Page 6 6

Table S4. Anisotropic displacement parameters $\left(\mathrm{A}^{2} \times 10^{3}\right)$ for $\left[\left(\mathrm{CH}_{3}\right)_{2} \mathrm{OCF}_{3}\right]^{+}\left[\mathrm{Sb}_{2} \mathrm{~F}_{11}\right]^{-}$. The anisotropic displacement factor exponent takes the form:

$-2 \mathrm{pi}^{2}\left[\mathrm{~h}^{2} \mathrm{a}^{* 2} \mathrm{U} 11+\ldots+2 \mathrm{hka} \mathrm{a}^{*} \mathrm{~b} * \mathrm{U12}\right]$

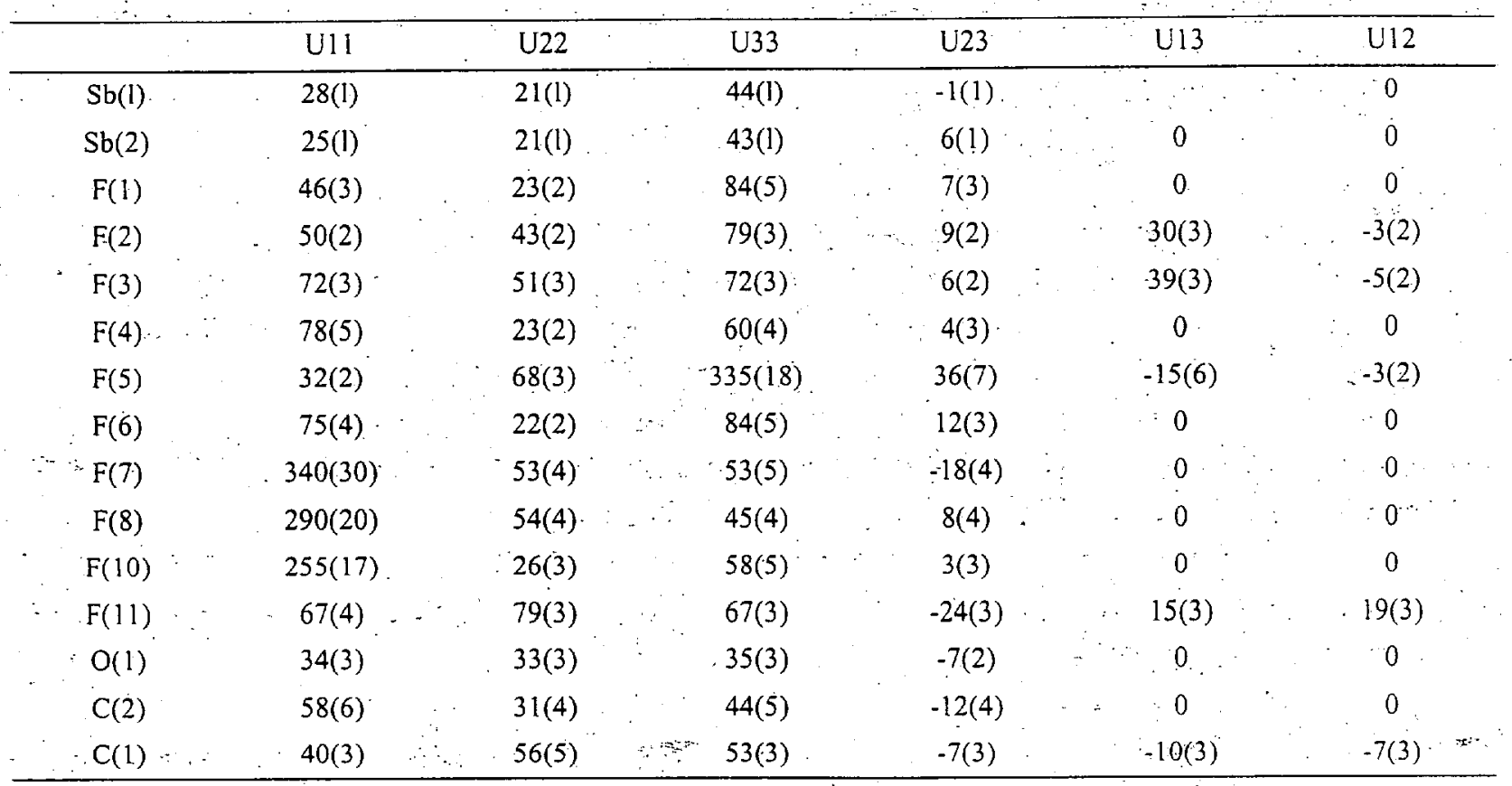


(c) 2001 American Chemical Society, Inorg. Chem., Minkwitz ic010239w Supporting Info Page 7 -

Table S5. Hydrogen coordinates $\left(\times 10^{4}\right)$ and isotropic displacement parameters $\left(\mathrm{A}^{2} \times 10^{3}\right)$ for $\left[\left(\mathrm{CH}_{3}\right)_{2} \mathrm{OCF}_{3}\right]^{+}\left[\mathrm{Sb}_{2} \mathrm{~F}_{11}\right]^{-}$

\begin{tabular}{|c|c|c|c|c|c|}
\hline . & $x$ & $y$ & $z$ & $\therefore$ & $U(e q)$ \\
\hline $\mathrm{H}(1)$ & 1434 & 5097 & 3594 & & 55 \\
\hline $\mathrm{H}(2)$ & 1303 & 6304 & 3227 & & 55 \\
\hline $\mathrm{H}(3)$ & 2172 & 5499 & 2452 & . & 55 \\
\hline
\end{tabular}

